\title{
Acute Respiratory Distress Syndrome Caused by Human Adenovirus in Adults: A Prospective Observational Study in Guangdong, 2019-2020
}

\section{Zhan Wu}

State Key Laboratory of Respiratory Disease

\section{Rong Zhang}

State Key Laboratory of Respiratory Disease

Dongdong Liu

State Key Laboratory of Respiratory Disease

Xuesong Liu

State Key Laboratory of Respiratory Disease

Jierong Zhang

State Key Laboratory of Respiratory Disease

\section{Zhihui Zhang}

State Key Laboratory of Respiratory Disease

Sibei Chen

State Key Laboratory of Respiratory Disease

\section{Weiqun He}

State Key Laboratory of Respiratory Disease

\section{Yimin Li}

State Key Laboratory of Respiratory Disease

\section{Yonghao Xu}

State Key Laboratory of Respiratory Disease

Xiaoqing Liu ( $\square$ lxq0703@126.com )

State Key Laboratory of Respiratory Disease

\section{Research}

Keywords: Acute respiratory distress syndrome, adenovirus, severe pneumonia, infection

Posted Date: July 13th, 2021

DOI: https://doi.org/10.21203/rs.3.rs-641948/v1 
License: (c) (i) This work is licensed under a Creative Commons Attribution 4.0 International License. Read Full License 


\section{Abstract}

Background: Viral causes of acute respiratory distress syndrome (ARDS) are mostly limited to influenza. However, adenovirus has been emerging as a cause of ARDS with a high mortality rate and described in adults are rare.

Methods: We conducted a prospective, single-center observational study of viral pneumonia with ARDS and confirmed adenovirus-associated ARDS in adults at our quaternary referral institution between March 2019 and June 2020. We prospectively analyzed clinical characteristics, laboratory test results, radiological characteristics, viral load from nasopharyngeal swabs and endotracheal aspirates, treatments, and outcomes for the study participants.

Results: The study enrolled 143 ARDS patients, including 47 patients with viral pneumonia-related ARDS, among which there were 14 adenovirus-associated ARDS patients, which accounted for $29.79 \%$ of the viral pneumonia-related ARDS cases. Among the adenovirus-associated ARDS patients, $78.57 \%$ were men with a mean age of $54.93 \pm 19.04$ years. Adenovirus-associated ARDS patients had no specific clinical characteristics, but they presented with shortness of breath and fever, and their initial chest radiographic findings were multifocal or showed diffuse opacity. The viral load and the positivity rate in the lower respiratory tract were higher than that of the upper respiratory tract in the patients with adenovirusassociated ARDS, and $85.71 \%$ of the patients had a significant decrease in the number of CD3+CD4+ T cells during the early stage. All patients required invasive mechanical ventilation treatment. The average time from shortness of breath to the application of invasive ventilation was 24 hours. The median duration of invasive mechanical ventilation was 22 days (14-75 days). Six patients (42.86\%) required renal replacement therapy, and three patients $(21.43 \%)$ required extracorporeal membrane oxygenation support. Additionally, $85.71 \%$ of the 14 adenovirus-associated ARDS patients survived.

Conclusion: Adenovirus infection is an important cause of virus-related ARDS. The positivity rate of adenovirus infection in lower respiratory tract secretions was higher than that in upper respiratory tract secretions in these patients. Most of the patients had a significant decrease in the number of CD3+CD4+ $T$ cells during the early stage. Early identification and intervention to prevent disease progression are essential for reducing the mortality rate in these patients.

\section{Background}

Acute respiratory distress syndrome (ARDS) is a rapid inflammatory lung injury with a high mortality rate that ranges from $30.0-46.0 \%$ [1-3]. Infectious pneumonia is the most common disease leading to ARDS, with viral infection accounting for approximately $22-40 \%$ of cases $[4,5]$. Influenza and rhinovirus have been most commonly detected in viral pneumonias followed by parainfluenza, adenovirus, respiratory syncytial virus, coronavirus, and human metapneumovirus $[4,6]$. However, the proportion of viral pneumonia leading to ARDS is unknown. Some viral infections, such as influenza A H1N1, H5N1, and H7N9 and the coronaviruses severe acute respiratory syndrome (SARS), severe acute respiratory 
syndrome coronavirus-2 (SARS-CoV-2), and Middle East Respiratory Syndrome (MERS), are associated with a high incidence of ARDS and increased mortality [7, 8]. An increased number of adenoviruses has been isolated from patients in recent years due to the development of molecular diagnostic technology [9]. Adenovirus-associated ARDS has been reported in adult patients with rapid progression to multi-organ failure and death, which has raised concerns. Additionally, there is limited information on ARDS and no consensus on its management [10].

Human adenoviruses (HAdVs) are non-enveloped DNA viruses that are associated with a wide range of clinical manifestations [11]. Adenovirus infection that causes severe fatal pneumonia has been well described in infants and children, but reports in adults are rare [12]. HAdV-associated ARDS infection is a devastating disease with rapid progression to multi-organ failure and death, and it can be fulminant with a mortality rate of approximately $40 \%$ in adult patients who require mechanical ventilation [13]. Patients with HAdV-associated ARDS have a poor survival rate.

Here, we performed a prospective, observational study to evaluate the proportion of viral pneumonia leading to ARDS in adult patients. We reported the cases patients with adenovirus-associated ARDS who required mechanical ventilation in the medical intensive care unit at our quaternary referral institution between November 2019 and December 2020. We analyzed clinical test results, laboratory test results, radiological characteristics, sequential viral load test results on nasopharyngeal swabs and endotracheal aspirates, treatments, and outcomes in these patients.

\section{Methods}

\section{Study design and population}

We performed a prospective, single-center observational study in adult patients with viral pneumonia with ARDS who were admitted to our respiratory intensive care unit (ICU) between March 2019 and June 2020. The study was approved by the Ethics Committee of the First Affiliated Hospital of Guangzhou Medical University. All patients provided written informed consent for their data to be used for research.

Severe ARDS was diagnosed in accordance with the Berlin definition [2], as follows: (1) developing within 1 week of a known clinical insult or new or worsening respiratory symptoms; (2) bilateral opacities not fully explained by effusions, lobar and/or lung collapse, or nodules; (3) respiratory failure not fully explained by cardiac failure or fluid overload; (4) partial oxygen pressure/fraction of inspired oxygen $\left(\mathrm{PaO}_{2} / \mathrm{FiO}_{2}\right) \leq 100 \mathrm{mmHg}$ with positive end-expiratory pressure (PEEP) $\geq 5 \mathrm{cmH}_{2} \mathrm{O}$; and (5) a chest radiograph with three or four quadrants with opacities. Patients with HAdV-55 infection and severe ARDS who failed conventional non-invasive positive pressure ventilation and invasive mechanical ventilation (IMV) were included in the analysis.

\section{Clinical data collection}


A standardized electronic case report form was used to prospectively collect the study data. The following data were obtained: age, sex, body mass index (BMI), preexisting comorbidities, Acute Physiology and Chronic Health Evaluation II (APACHEII) score, daily Sequential Organ Failure Assessment (SOFA) score, and clinical pulmonary infection score (CPIS). Clinical symptoms (fever, cough, sputum, and dyspnea), signs (body temperature, heart rate, respiratory frequency, and blood pressure), and laboratory data (procalcitonin, white blood cell count, lymphocyte, platelet count, and creatinine level), microbiological findings, and images of the lung (chest X-ray and computed tomography) were also collected. Additionally, complications, treatments, respiratory support, and clinical outcomes were recorded.

\section{Molecular assay for respiratory virus detection}

Nasopharyngeal swabs and endotracheal aspirates (ETA) were collected at admission and during hospitalization and stored at $-80^{\circ} \mathrm{C}$ until testing. All nasopharyngeal swabs and ETA samples were analyzed using multiplex quantitative polymerase chain reaction (Q-PCR) technology. This assay tested for 24 respiratory viruses (adenovirus; influenza virus type A H1N1, H3N2, H1N1 2009, H5N1, and H7N9; influenza virus type $B$; respiratory syncytial virus types $A$ and $B$; parainfluenza viruses type $1,2,3$, and 4; metapneumovirus; coronavirus OC43, 229E, NL63, HKU1, SARS, and MERS; rhinovirus; bocavirus; Nipah virus; and circovirus).

The viral load was indicated as the cycle threshold (Ct) value. Ct values were used to analyze each patient's viral load. Ct values were inversely correlated to the quantity of RNA target present in the specimen. A Ct value of $<40$ was defined as positive for the viruses and $>40$ was defined as a negative result. Samples with a Ct value between 37 and 40 were retested at least twice.

\section{Statistical analysis}

Measurement variables were summarized using the median (interquartile range) or the mean and range, and enumeration variables were presented using the frequency and percentage. The Student's $t$-test or the Mann-Whitney U-test was used to compare the measurement variables, and $p<0.05$ was considered to be significant. Statistical analysis was performed using GraphPad Prism 7.0.

\section{Results}

The study included 143 patients, including 47 patients (32.87\%) with viral pneumonia-related ARDS and 14 adenovirus-positive ARDS patients, which accounted for $29.79 \%$ of viral pneumonia-related ARDS (Fig. 1).

The general condition and clinical characteristics of the patients with adenovirus-associated ARDS are shown in Table 1. The mean age was $54.93 \pm 19.04$ years, and $78.57 \%$ were males, $50 \%$ were smokers, and $71.43 \%$ had high nutritional risk (Table 1 ). In addition, $28.57 \%$ of the patients had concurrent lung diseases, and $28.57 \%$ of the patients also had an immunodeficiency. All patients had cough, sputum expectoration, and shortness of breath, and $92.86 \%$ of them had a fever. Most patients presented with a 
low-grade fever. As shown in Table 2, the $\mathrm{PaO}_{2} / \mathrm{FiO}_{2}$ for $64.29 \%$ of the patients was between 150 and 200 $\mathrm{mmHg}$. Most of the patients had normal blood $\mathrm{CO}_{2}$, manifesting as type I respiratory failure. The average time from the onset of shortness of breath to tracheal intubation was 24 hours. Hypoproteinemia occurred in $85.71 \%$ of the patients, and abnormal coagulation function occurred in $>60 \%$ of the patients. At the time of enrollment, $64.29 \%$ of the patients had a low total number of white blood cells, and more than $70 \%$ of the patients had a decrease in the total number and percentage of lymphocytes. Additionally, $85.71 \%$ of the patients had a significant decrease in the number of CD $3+C D 4+T$ cells during the early stage and an increase in interleukin (IL)- 6 levels. Most of the patients had diffuse infiltrates in both lungs. Three $(21.43 \%)$ of the patients had interstitial abnormalities. Five patients $(35.71 \%)$ had pleural effusions. Only a few patients showed ground-glass opacity (Fig. 2). 
Table 1

Demographic data and clinical characteristics of the patients with adenovirus-associated ARDS

\begin{tabular}{|c|c|}
\hline & Mean \pm Standard Deviation, $n(\%)$ \\
\hline Sex(male) & $11(78.57 \%)$ \\
\hline Age(years) & $54.93 \pm 19.04$ \\
\hline $\mathrm{BMI}\left(\mathrm{kg} / \mathrm{m}^{2}\right)$ & $20.30 \pm 3.03$ \\
\hline APACHEII Score & $19.14 \pm 7.35$ \\
\hline SOFA Score & $10.50 \pm 4.09$ \\
\hline CPIS Score & $6.50 \pm 1.15$ \\
\hline Smoking & $7(50 \%)$ \\
\hline Nutrition Score & $5.64 \pm 2.31$ \\
\hline High Nutritional Risk & $9(64.29 \%)$ \\
\hline \multicolumn{2}{|l|}{ Underlying lung disease } \\
\hline COPD & $2(14.28 \%)$ \\
\hline Bronchiectasis & $1(7.14 \%)$ \\
\hline Interstitial lung disease & $1(7.14 \%)$ \\
\hline Immunodeficiency & $4(28.57 \%)$ \\
\hline \multicolumn{2}{|l|}{ Symptoms } \\
\hline Fever & $13(92.86 \%)$ \\
\hline Cough & $14(100.00 \%)$ \\
\hline Shortness of breath & $14(100.00 \%)$ \\
\hline \multicolumn{2}{|l|}{ Temperature $\left({ }^{\circ} \mathrm{C}\right)$} \\
\hline $36.0-37.2$ & $7(50 \%)$ \\
\hline $37.3-38.0$ & $5(35.71 \%)$ \\
\hline $38.1-39.0$ & $1(7.14 \%)$ \\
\hline $39.1-41$ & $1(7.14 \%)$ \\
\hline Heart rate(bpm) & $98.36 \pm 24.11$ \\
\hline Respiratory Rate(bpm) & $22.93 \pm 6.96$ \\
\hline $\mathrm{MAP}(\mathrm{mmHg})$ & $75.86 \pm 12.27$ \\
\hline
\end{tabular}




\begin{tabular}{|c|c|}
\hline & Mean \pm Standard Deviation,n(\%) \\
\hline \multicolumn{2}{|l|}{ Co-infection } \\
\hline \multicolumn{2}{|l|}{ Other viruses } \\
\hline Influenza Virus & $2(14.29 \%)$ \\
\hline Human Coronavirus OC43 & $1(7.14 \%)$ \\
\hline Human Rhinovirus & $1(7.14 \%)$ \\
\hline Bacteria & $10(71.43 \%)$ \\
\hline Acinetobacter baumannii & $6(42.86 \%)$ \\
\hline Stenotrophomonas Maltophilia & $3(21.43 \%)$ \\
\hline Klebsiella pneunoniae & $2(14.29 \%)$ \\
\hline Other bacteria & $4(28.57 \%)$ \\
\hline
\end{tabular}


Table 2

Laboratory findings and radiographic results for patients with adenovirusassociated ARDS

\begin{tabular}{|c|c|}
\hline Characteristic & Mean \pm Standard Deviation,n(\%) \\
\hline White blood cell count, $\times 10 \otimes / L$ & $12.66 \pm 6.40$ \\
\hline$<4$ & 0 \\
\hline$>10$ & $9(64.29 \%)$ \\
\hline Platelet count,$\times 10 \otimes / L$ & $165.43 \pm 103.26$ \\
\hline$\otimes 100$ & $5(35.71 \%)$ \\
\hline Lymphocyte, $\times 10 \rrbracket / L$ & $0.64 \pm 0.49$ \\
\hline$\varangle 0.9$ & $10(71.43 \%)$ \\
\hline CD3 + CD45 + T, Cell/UL & $278.00(171.50-634.00)$ \\
\hline 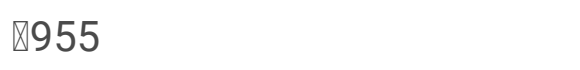 & $12(85.71 \%)$ \\
\hline $\mathrm{CD} 3+\mathrm{CD} 4+\mathrm{T}, \mathrm{Cell} / \mathrm{UL}$ & $148.00(106.00-365.00)$ \\
\hline$\varangle 550$ & $12(85.71 \%)$ \\
\hline CD3 + CD8 + T, Cell/UL & $120.00(57.50-282.00)$ \\
\hline$\otimes 320$ & $11(78.57 \%)$ \\
\hline Interleukin-6,pg/ml & $39.37(12.53-143.06)$ \\
\hline$₫ 5.3$ & $12(85.71 \%)$ \\
\hline Procalcitonin, $\mathrm{ng} / \mathrm{mL}$ & $0.38(1.90-4.66)$ \\
\hline$\varangle 0.05$ & $14(100.00 \%)$ \\
\hline \multicolumn{2}{|l|}{ Arterial blood gas analysis: } \\
\hline $\mathrm{pH}$ & $7.37 \pm 0.09$ \\
\hline $\mathrm{pCO}_{2}, \mathrm{mmHg}$ & $44.12 \pm 6.83$ \\
\hline $\mathrm{P} / \mathrm{F}$ & $168.59 \pm 41.77$ \\
\hline$\otimes 150$ & $5(35.71 \%)$ \\
\hline $150-200$ & $9(64.29 \%)$ \\
\hline Albumin, $\mathrm{g} / \mathrm{L}$ & $30.96 \pm 10.14$ \\
\hline$\varangle 35$ & $12(85.71 \%)$ \\
\hline Prothrombin time, s & $16.06 \pm 2.73$ \\
\hline
\end{tabular}




\begin{tabular}{|ll|}
\hline Characteristic & Mean \pm Standard Deviation, $\mathrm{n}(\%)$ \\
\hline$\otimes 14.5$ & $10(71.43 \%)$ \\
\hline Activated partial thromboplastin time,s & $51.23 \pm 28.21$ \\
\hline $\mathbf{4} 2.8$ & $9(64.29 \%)$ \\
\hline Creatinine, umol/L & $162.55(87.50-282.50)$ \\
\hline 133 & $9(64.29 \%)$ \\
\hline Abnormalities on chest radiograph & \\
\hline Ground-glass opacity & $2(14.29 \%)$ \\
\hline Bilateral patchy shadowing & $14(100 \%)$ \\
\hline Interstitial abnormalities & $3(21.43 \%)$ \\
\hline Pleural effusions & $5(35.71 \%)$ \\
\hline
\end{tabular}

Within 48 hours of enrollment, paired upper respiratory tract (URT) samples (nasopharyngeal swab) and lower respiratory tract (LRT) samples (sputum and bronchoalveolar lavage) were collected from the patients to detect the virus using multiplex Q-PCR. As shown in Table 3, the LRT samples showed a significantly higher positive rate $(100 \%, 14 / 14)$ than URT samples $(64.29 \%, 9 / 14)$ for detecting respiratory viruses $(p<0.001)$, and LRT samples had higher levels of detection using a Ct value of $31.66(20.56-$ 34.78) compared with URT sampling using a Ct value of 39.14 (27.95-40) (Fig. 3). On average, URT sites cleared faster than LRT sites (Fig. 4). Detection of other viruses, fungi, and bacteria from the sputum collected from patients within 48 hours of enrollment showed that four patients $(28.57 \%)$ also had another viral infection, and among these patients, the most common virus was the influenza virus that accounted for $14.29 \%$ of viral infections. Nine patients $(64.29 \%)$ had positive bacterial cultures in the sputum, with the highest positivity rate for Acinetobacter baumannii, which was detected in six patients (42.86\%). All patients had a negative fungal culture from the sputum, blood, and bronchoalveolar lavage fluid in (1-3)- $\beta$-D-glucan (G) and galactomannan (GM) tests.

Table 3

Comparison of multiplex Q-PCR results between URT and LRT samples

\begin{tabular}{|llll|}
\hline & URT & LRT & P value \\
\hline Detected n (\%) & $9(64.29 \%)$ & $14(100 \%)$ & $<0.001$ \\
\hline Undetected n (\%) & $5(35.71 \%)$ & 0 & $<0.001$ \\
\hline Time of virus clearance(days) & $15.16(4-37)$ & $24.17(6-42)$ & $<0.001$ \\
\hline
\end{tabular}

All patients received antiviral therapy, including ganciclovir ( $5 \mathrm{mg} / \mathrm{kg}$ intravenously every 12 hours) and oseltamivir. Considering that bacterial coinfection may combine with a severe viral infection, broad- 
spectrum intravenous antibiotics were administered to all patients. Additionally, $78.57 \%$ of the patients received immunoglobulin. All patients required invasive ventilation, and the median duration of mechanical ventilation was 22 days (interquartile range [IQR], 14-75 days). Three patients (21.43\%) required extracorporeal membrane oxygenation (ECMO), with a treatment duration of 7 to 12 days, and one patient $(7.14 \%)$ underwent prone position ventilation. The average length of ICU stay was 26.50 days (IQR, 15-75 days), and the median length of hospital stay was 37.5 days (IQR, 29.75-81.00 days). Among the 14 patients in this study, there were two deaths, with a mortality rate of $14.29 \%$ during the study period (Table 4).

Table 4

Complications, treatments, and clinical outcomes in patients with adenovirus-associated ARDS

\begin{tabular}{|ll|}
\hline Characteristic & No(\%)/Median(Interquartile range) \\
\hline Complications & $10(71.43 \%)$ \\
\hline AKI & $4(28.57 \%)$ \\
\hline Sepsis shock & $2(14.29 \%)$ \\
\hline Pneumothorax & \\
\hline Treatments & $6(42.86 \%)$ \\
\hline CRRT & $3(21.43 \%)$ \\
\hline ECMO & $1(7.14 \%)$ \\
\hline Prone position ventilation & $11(78.57 \%)$ \\
\hline Vasoactive & $11(78.57 \%)$ \\
\hline Muscle relaxants & $6(42.86 \%)$ \\
\hline Intravenous immune globulin & \\
\hline anti-viral agents & $7(50.00 \%)$ \\
\hline Oseltamivir & $11(78.57 \%)$ \\
\hline Ganciclovir & $26.50(15.75-75.25)$ \\
\hline Clinical outcomes & $1.00(1.00-2.00)$ \\
\hline Duration of dyspnea to IMV & $22.00(14.00-75.25)$ \\
\hline Duration of IMV(days) & \\
\hline Length of ICU stay(days) & $26.75-81.00)$ \\
\hline Length of hospital stay(days) & $37.29 \%)$ \\
\hline Death & \\
\hline
\end{tabular}




\section{Discussion}

The number of ARDS cases caused by viral pneumonia is increasing, resulting in a high mortality rate [14]. Previous studies have mostly focused on influenza viruses, such as $H 1 N 1$ and $H 7 N 9[15,16]$. Cases of adenovirus-associated ARDS have gradually been increasing, which may be due to recent developments in molecular diagnostic technology [17]. However, there is limited data about the viral etiology of ARDS in patients who required mechanical ventilation. Zhou et al. reported that the incidence of adenovirus pneumonia ranks third among viral pneumonia in adults in China [6]. They have also found that among virus-related ARDS patients with $\mathrm{PO}_{2} / \mathrm{FiO}_{2}$ ratio $<200 \mathrm{mmHg}, \mathrm{HAdV}$ infection was the most frequently detected virus [6]. Our study showed that in all ARDS patients, virus-related ARDS accounted for $32.87 \%$ of infections. Among these infections, adenovirus-associated ARDS accounted for $9.79 \%$ of all ARDS patients and $29.79 \%$ of virus-related ARDS patients. The prevalence of respiratory viruses varies in different countries and different populations $[4-6,13]$. To the best of our knowledge, this is the first study about the viral etiology in ARDS patients who required mechanical ventilation in China.

There are few studies on adenovirus-associated ARDS in adults who required mechanical ventilation [46 , 13]. In 2000, two non-immunocompromised soldiers became infected with adenovirus, which resulted in ARDS $[4-6,13]$. In 2006, there was an outbreak of adenovirus pneumonia caused by HAdV-B11 in the USA; 140 people were diagnosed with HAdV infection, and 24 patients who were diagnosed with ARDS were admitted to the ICU [22]. Some studies have proposed that severe adenovirus infection is likely to occur in children and immunocompromised adults, such as HIV patients, and patients after transplantation [23, 24]. In this study, most of the adenovirus-associated ARDS patients, with a mean age of 54 years, had no underlying diseases. Among these patients, $78.57 \%$ of them were men, and $50 \%$ of the patients were smokers, suggesting that severe adenovirus pneumonia in non-immunocompromised adults was likely to occur in middle-aged men. Additionally, $85.71 \%$ of the patients had a significant decrease in the number of $\mathrm{CD} 3+\mathrm{CD} 4+\mathrm{T}$ cells during the early stage, which suggests that adenovirus infection may cause immune system dysregulation.

Delayed clearance of respiratory adenovirus infection leads to a worse prognosis in these patients, and monitoring the viral load may help to predict the disease severity and the patients' prognosis $[25,26]$. Rapid identification of adenovirus viral infection is critical to reduce the overall costs of patient management. Multiplex Q-PCR is of great value in the early diagnosis of virus infection because of its high sensitivity $[27,28]$. However, viral testing of URT and LRT samples may yield different results [27, 28]. Currently, few studies have been published that compare the diagnostic yields of URT and LRT samples to detect adenovirus. In our study, the detection rates of adenovirus from LRT and URT samples were $100 \%$ and $64.29 \%$, respectively. Similarly, a European study in 2016 reported that the overall virus positivity rate of URT was lower than that of the LRT specimens ( $24.5 \% \mathrm{vs.} 44.2 \%)$ [30]. In this study, the percentage of positive specimens was higher in LRT than in URT specimens. On average, URT specimens cleared faster than LRT specimens, suggesting that traditional nasopharyngeal diagnostic techniques can miss cases of severe adenovirus infection. This suggests that LRT specimens are more reliable for 
diagnosing severe adenovirus infection, especially in patients with pneumonia that occurs several days after the infection onset when the frequency of virus detection in the URT has already decreased.

Several studies have shown that the mortality of severe adenovirus-associated ARDS can be as high as $26.7-80 \%$ in adults [10]. In our study, among the 14 patients with adenovirus-associated ARDS, there were only two deaths, and the mortality rate was $14.29 \%$. Compared with previous studies, the mortality rate of adenovirus-associated ARDS in this study was relatively low, and this may have several explanations. First, rapid identification of adenovirus viral infection and early intervention are important to reduce the overall mortality rate. In this study, the time from onset to intubation was relatively short. In addition, the $\mathrm{PO}_{2} / \mathrm{FiO}_{2}$ ratio for most adenovirus-associated ARDS patients was $>150$ in this study, while most other studies showed that the $\mathrm{PO}_{2} / \mathrm{FiO}_{2}$ ratio in patients with severe pneumonia was $<150[6,23$, 31]. Considering that the condition of severe adenovirus pneumonia patients was more advanced, the above results suggested that early intervention in adenovirus pneumonia-related ARDS helped to improve the patients' prognosis. Second, establishing organ support, such as application of early renal replacement treatment and ECMO are important. Adenovirus-associated ARDS completely resolved in three patients who were supported by ECMO in this study, suggesting that early application of ECMO improved the prognosis of patients with adenovirus-associated ARDS. Finally, timely initiation of antiviral therapy is very important to improve patient outcome. Currently, antiviral therapies for adenovirus infection remain controversial. No specific and effective antiviral drug is available for adenovirus infection [32]. Some studies have shown that cidofovir antiviral therapy in severe adenovirus pneumonia improves the clinical prognosis [33]. However, clinical application of cidofovir is limited due to its toxic side effects and low-quality evidence. Other case reports have also shown that ribavirin can be used to treat adenovirus infection [34,35]. Ganciclovir has been shown to be effective for treating adenovirus infection in animal experiments [36]. In our study, 11 patients (78.57\%) received antiviral therapy with ganciclovir and immunoglobulin therapy after confirming adenovirus infection, which might be the reason for the lower mortality.

This study prospectively observed the viral etiology of ventilated ARDS patients, especially for patients where adenovirus was associated with ARDS. We compared the adenovirus detection rate and adenovirus load in different respiratory tract specimens, which were also a highlight of the study. This study also has several limitations. It was a single-center study with a relatively small number of patients enrolled. In addition, this study used multiplex Q-PCR to detect multiple respiratory viruses at the same time without genotyping the adenovirus.

\section{Conclusions}

Our findings indicated that adenovirus infection was an important cause of viral-related ARDS. The detection rate of adenovirus from the LRT was higher than that from the URT. However, mortality due to adenovirus-associated ARDS was high. Therefore, rapid identification of severe adenovirus infection, early effective intervention, and timely initiation of antiviral therapy are essential to reduce mortality and improve the prognosis in patients with adenovirus-associated ARDS. 


\section{Abbreviations}

ARDS

Acute respiratory distress syndrome

ECMO

Extracorporeal membrane oxygenation

SARS

severe acute respiratory syndrome

SARS-CoV-2

Severe acute respiratory syndrome coronavirus2

MERS

Middle East Respiratory Syndrome

HAdVs

Human adenoviruses

AKI

Acute kidney injury

CRRT

Continuous renal replacement therapy

PEEP

Positive end-expiratory pressure

IMV

Invasive mechanical ventilation

BMI

Body mass index

APACHEII

Acute Physiology and Chronic Health Evaluation II

SOFA

Sequential Organ Failure Assessment

CPIS

Clinical pulmonary infection score

ETA

Endotracheal aspirates

PCR

Quantification polymerase chain reaction

Ct

Cycle threshold

URT

Upper respiratory tract

LRT

Lower respiratory tract 


\section{Declarations}

\section{Ethics approval and consent to participate}

The study was approved by the local research ethics committee (2019-19), which waived the need for informed consent for the retrospective collection of demographic, physiological, and hospital outcome data, based on Chinese legislation.

\section{Consent for publication}

Not applicable.

\section{Availability of data and material}

All data generated and/or analyzed during this study are included in this published article and its supplementary information files.

\section{Competing interests}

The authors declare that they have no competing interests.

\section{Funding}

The study was supported by National Natural Science Foundation of China, Grant Nos. 82070084(XQL), 81700080 (RZ) and by National Science and Technology Major Project (No. 2017ZX10204401003).

\section{Authors' contributions}

Study conception and design: XL, Data collection: ZW, RZ, DL, XL, SC, YX, JZ and ZZ. Analysis and interpretation: $\mathrm{ZW}, \mathrm{RZ}, \mathrm{WH}, \mathrm{XL}$ and $\mathrm{YL}$. Writing the manuscript: $\mathrm{RZ}$ and $\mathrm{ZW}$. All authors read and approved the final manuscript.

\section{Acknowledgement}

We thank Jodi Smith, PhD ELS, from Liwen Bianji (Edanz) (www.liwenbianji.cn/ac) for editing the English text of a draft of this manuscript.

\section{References}

1. Thompson BT, Chambers RC, Liu KD. Acute Respiratory Distress Syndrome. N Engl J Med. 2017;377(6):562-72.

2. Confalonieri M, Salton F, Fabiano F. Acute respiratory distress syndrome. Eur Respir Rev 2017, 26(144). 
3. Chiumello D, Brochard L, Marini JJ, Slutsky AS, Mancebo J, Ranieri VM, Thompson BT, Papazian L, Schultz MJ, Amato M, et al. Respiratory support in patients with acute respiratory distress syndrome: an expert opinion. CRIT CARE. 2017;21(1):240.

4. Jain S, Self WH, Wunderink RG, Fakhran S, Balk R, Bramley AM, Reed C, Grijalva CG, Anderson EJ, Courtney DM, et al. Community-Acquired Pneumonia Requiring Hospitalization among U.S. Adults. N Engl J Med. 2015;373(5):415-27.

5. Kalil AC, Thomas PG. Influenza virus-related critical illness: pathophysiology and epidemiology. CRIT CARE. 2019;23(1):258.

6. Zhou F, Wang Y, Liu Y, Liu X, Gu L, Zhang X, Pu Z, Yang G, Liu B, Nie Q, et al: Disease severity and clinical outcomes of community-acquired pneumonia caused by non-influenza respiratory viruses in adults: a multicentre prospective registry study from the CAP-China Network. EUR RESPIR J 2019, $54(2)$.

7. Li X, Ma X: Acute respiratory failure in COVID-19: is it "typical" ARDS? CRIT CARE 2020, 24(1):198.

8. Herold S, Becker C, Ridge KM, Budinger GR. Influenza virus-induced lung injury: pathogenesis and implications for treatment. EUR RESPIR J. 2015;45(5):1463-78.

9. Cao B, Huang GH, Pu ZH, Qu JX, Yu XM, Zhu Z, Dong JP, Gao Y, Zhang YX, Li XH, et al. Emergence of community-acquired adenovirus type 55 as a cause of community-onset pneumonia. CHEST. 2014;145(1):79-86.

10. Sun B, He H, Wang Z, Qu J, Li X, Ban C, Wan J, Cao B, Tong Z, Wang C. Emergent severe acute respiratory distress syndrome caused by adenovirus type 55 in immunocompetent adults in 2013: a prospective observational study. CRIT CARE. 2014;18(4):456.

11. Binder AM, Biggs HM, Haynes AK, Chommanard C, Lu X, Erdman DD, Watson JT, Gerber SI. Human Adenovirus Surveillance - United States, 2003-2016. MMWR Morb Mortal Wkly Rep. 2017;66(39):1039-42.

12. Wong-Chew RM, García-León ML, Noyola DE, Perez GL, Gaitan MJ, Vilaseñor-Sierra A, MartinezAguilar G, Rivera-Nuñez VH, Newton-Sánchez OA, Firo-Reyes V, et al. Respiratory viruses detected in Mexican children younger than 5 years old with community-acquired pneumonia: a national multicenter study. INT J INFECT DIS. 2017;62:32-8.

13. Vashisht R, Mirzai S, Koval C, Duggal A. Adenovirus-associated Acute Respiratory Distress Syndrome: Need for a Protocol-based Approach. Indian J Crit Care Med. 2020;24(5):367-8.

14. Matthay MA, Aldrich JM, Gotts JE. Treatment for severe acute respiratory distress syndrome from COVID-19. Lancet Respir Med. 2020;8(5):433-4.

15. Chen J, Hu C, Chen L, Tang L, Zhu Y, Xu X, Chen L, Gao H, Lu X, Yu L, et al. Clinical Study of Mesenchymal Stem Cell Treatment for Acute Respiratory Distress Syndrome Induced by Epidemic Influenza A (H7N9) Infection: A Hint for COVID-19 Treatment. Engineering (Beijing). 2020;6(10):1153-61.

16. Tang X, Du RH, Wang R, Cao TZ, Guan LL, Yang CQ, Zhu Q, Hu M, Li XY, Li Y, et al: Comparison of Hospitalized Patients With ARDS Caused by COVID-19 and H1N1. CHEST 2020, 158(1):195-205. 
17. Cha MJ, Chung MJ, Lee KS, Kim TJ, Kim TS, Chong S, Han J. Clinical Features and Radiological Findings of Adenovirus Pneumonia Associated with Progression to Acute Respiratory Distress Syndrome: A Single Center Study in 19 Adult Patients. KOREAN J RADIOL. 2016;17(6):940-9.

18. Ryu JS, Cho JH, Han HS, Jung MH, Yoon YH, Song ES, Lee JY, Kim SY, Lee KW, Kwak SM, et al. Acute respiratory distress syndrome induced by adenovirus in an otherwise healthy woman. YONSEI MED J. 2003;44(4):732-5.

19. Narra R, Bono P, Zoccoli A, Orlandi A, Piconi S, Grasselli G, Crotti S, Girello A, Piralla A, Baldanti F, et al. Acute respiratory distress syndrome in adenovirus type 4 pneumonia: A case report. J CLIN VIROL. 2016;81:78-81.

20. Pfortmueller CA, Barbani MT, Schefold JC, Hage E, Heim A, Zimmerli S. Severe acute respiratory distress syndrome (ARDS) induced by human adenovirus B21: Report on 2 cases and literature review. J CRIT CARE. 2019;51:99-104.

21. Two fatal cases of adenovirus-related illness in previously healthy young adults-Illinois, 2000. MMWR Morb Mortal Wkly Rep 2001, 50(26):553-555.

\section{Acute respiratory disease associated with adenovirus serotype 14-four states, 2006-2007. MMWR} Morb Mortal Wkly Rep 2007, 56(45):1181-1184.

23. Chen X, Lv J, Qin L, Zou C, Tang L. Severe Adenovirus Pneumonia Requiring Extracorporeal Membrane Oxygenation Support in Immunocompetent Children. FRONT PEDIATR. 2020;8:162.

24. Engelmann I, Coiteux V, Heim A, Magro L, Dewilde A, Dulery R, Hober D, Yakoub-Agha I. Severe Adenovirus Pneumonia Followed by Bacterial Septicaemia: Relevance of Co-Infections in Allogeneic Hematopoietic Stem Cell Transplantation. Infect Disord Drug Targets. 2016;16(1):69-76.

25. Hafidi M, Janin-Manificat H, Denis P, Charleux B, Rabilloud M, Boibieux A, Burillon C, Kodjikian L, Frobert E. Acute Retinal Necrosis: Virological Features Using Quantitative Polymerase Chain Reaction, Therapeutic Management, and Clinical Outcomes. AM J OPHTHALMOL. 2019;208:376-86.

26. Huh K, Kim I, Jung J, Lee JE, Jhun BW, Gu SH, Song DH, Lee EY, Jeong HJ, Yoo H. Prolonged shedding of type 55 human adenovirus in immunocompetent adults with adenoviral respiratory infections. Eur J Clin Microbiol Infect Dis. 2019;38(4):793-800.

27. Albert E, Torres I, Bueno F, Huntley D, Molla E, Fernández-Fuentes M, Martínez M, Poujois S, Forqué L, Valdivia A, et al. Field evaluation of a rapid antigen test (Panbio ${ }^{\mathrm{TM}}$ COVID-19 Ag Rapid Test Device) for COVID-19 diagnosis in primary healthcare centres. Clin Microbiol Infect. 2021;27(3):472-7.

28. Paiva JA, Laupland KB. Real -time PCR for early microbiological diagnosis: is it time? Intensive Care Med. 2017;43(11):1714-6.

29. Robert S, Lhommet C, Le Brun C, Garot D, Legras A, Mankikian J, Goudeau A. Diagnostic performance of multiplex PCR on pulmonary samples versus nasopharyngeal aspirates in community-acquired severe lower respiratory tract infections. J CLIN VIROL. 2018;108:1-5.

30. Boerger AC, Binnicker MJ. Comparison of the Panther Fusion respiratory panels to routine methods for detection of viruses in upper and lower respiratory tract specimens. Diagn Microbiol Infect Dis. 2020;97(2):115014. 
31. Shen Y, Cai G, Gong S, Dong L, Yan J, Cai W. Interaction between low tidal volume ventilation strategy and severity of acute respiratory distress syndrome: a retrospective cohort study. CRIT CARE. 2019;23(1):254.

32. Lynch JR, Kajon AE. Adenovirus: Epidemiology, Global Spread of Novel Serotypes, and Advances in Treatment and Prevention. Semin Respir Crit Care Med. 2016;37(4):586-602.

33. Ko JH, Lim JU, Choi JY, Oh HS, Yoo H, Jhun BW, Huh K, Peck KR. Early cidofovir administration might be associated with a lower probability of respiratory failure in treating human adenovirus pneumonia: a retrospective cohort study. Clin Microbiol Infect. 2020;26(5):646-9.

34. Maslo C, Girard PM, Urban T, Guessant S, Rozenbaum W. Ribavirin therapy for adenovirus pneumonia in an AIDS patient. Am J Respir Crit Care Med. 1997;156(4 Pt 1):1263-4.

35. Yoon BW, Song YG, Lee SH. Severe community-acquired adenovirus pneumonia treated with oral ribavirin: a case report. BMC Res Notes. 2017;10(1):47.

36. Ying B, Tollefson AE, Spencer JF, Balakrishnan L, Dewhurst S, Capella C, Buller RM, Toth K, Wold WS. Ganciclovir inhibits human adenovirus replication and pathogenicity in permissive immunosuppressed Syrian hamsters. Antimicrob Agents Chemother. 2014;58(12):7171-81.

\section{Figures}

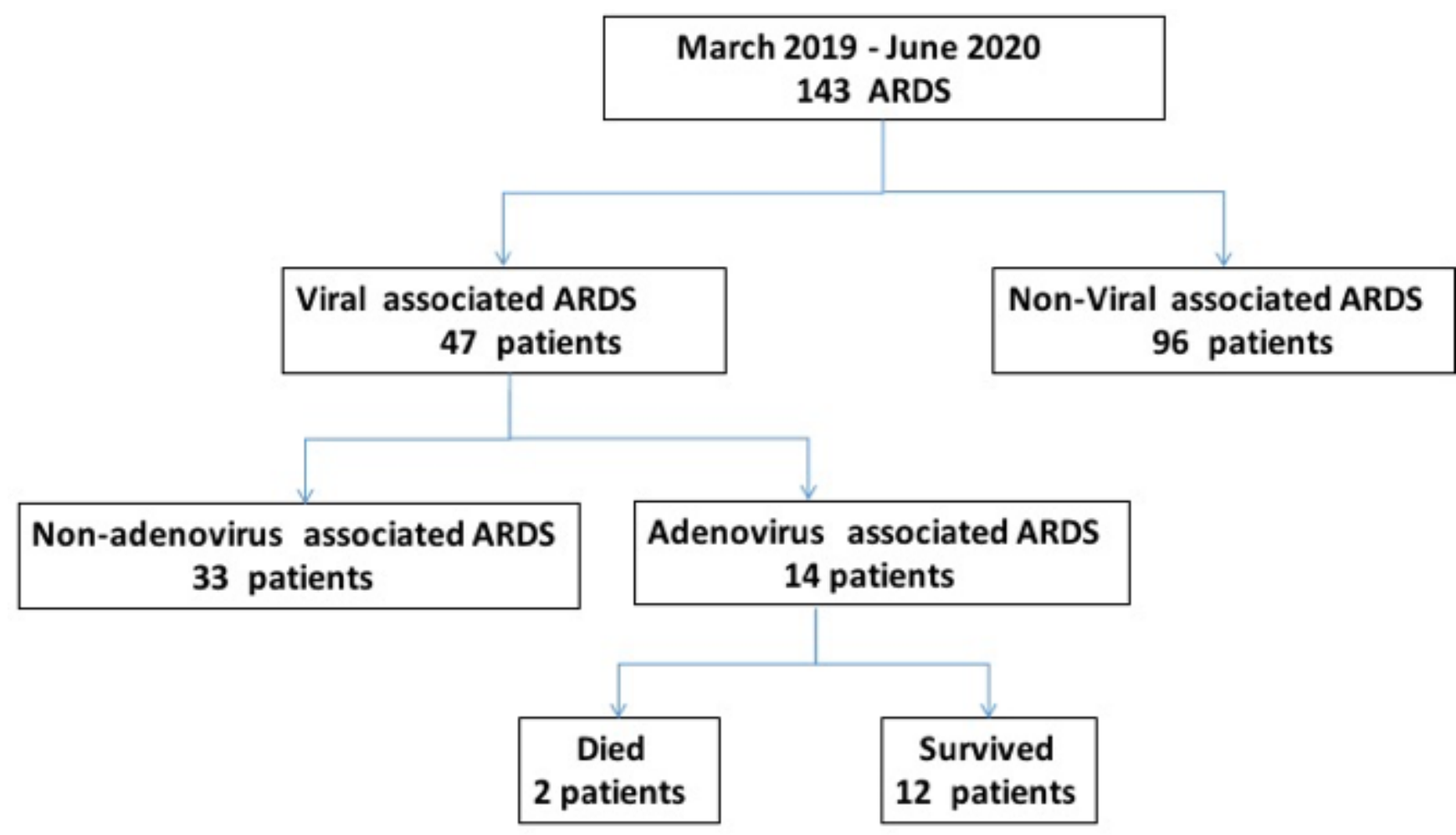

Figure 1

Flowchart of patient groups in the study. ARDS, acute respiratory distress syndrome 

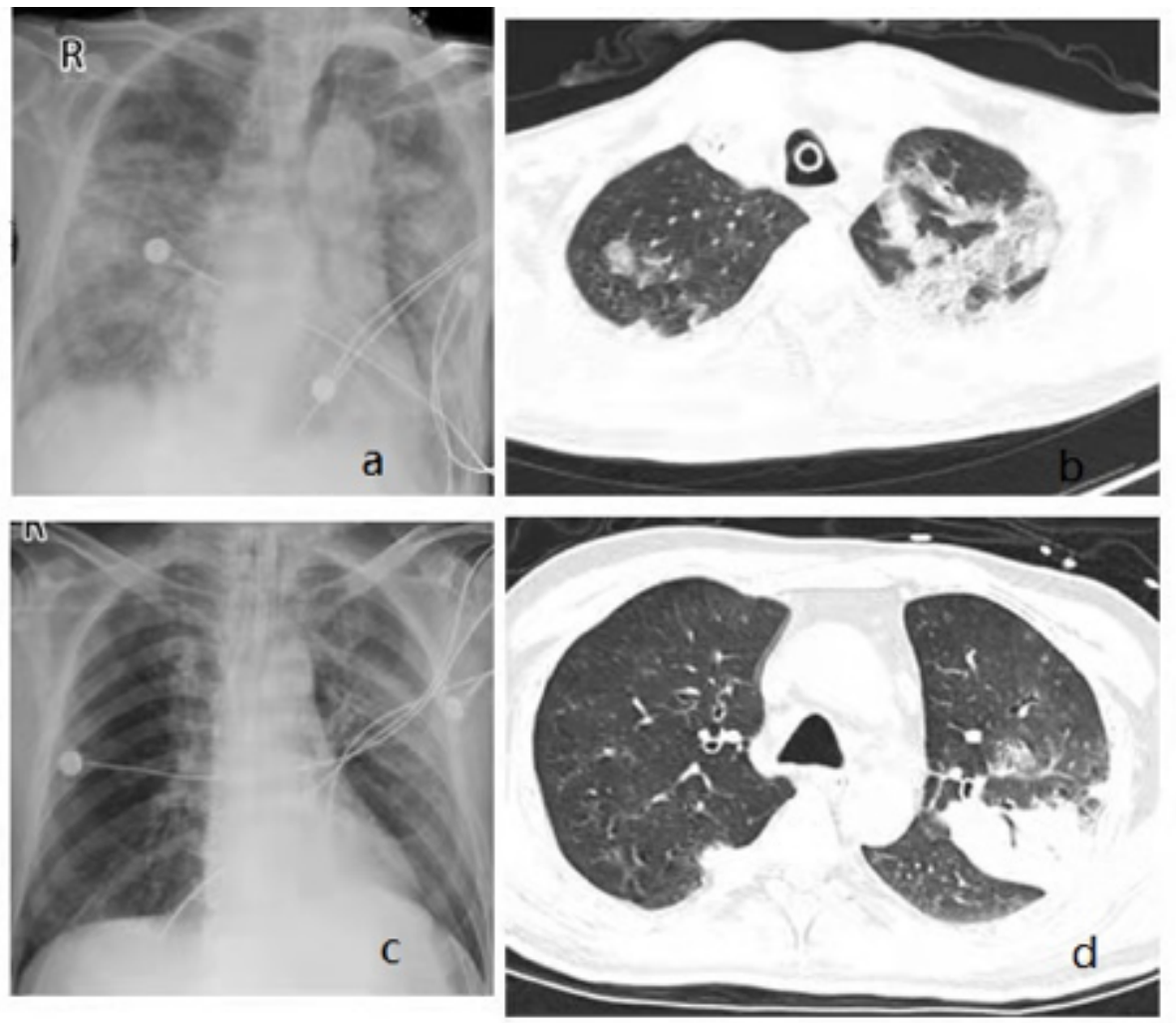

Figure 2

Imaging findings showing diffuse multifocal or diffuse opacity. 


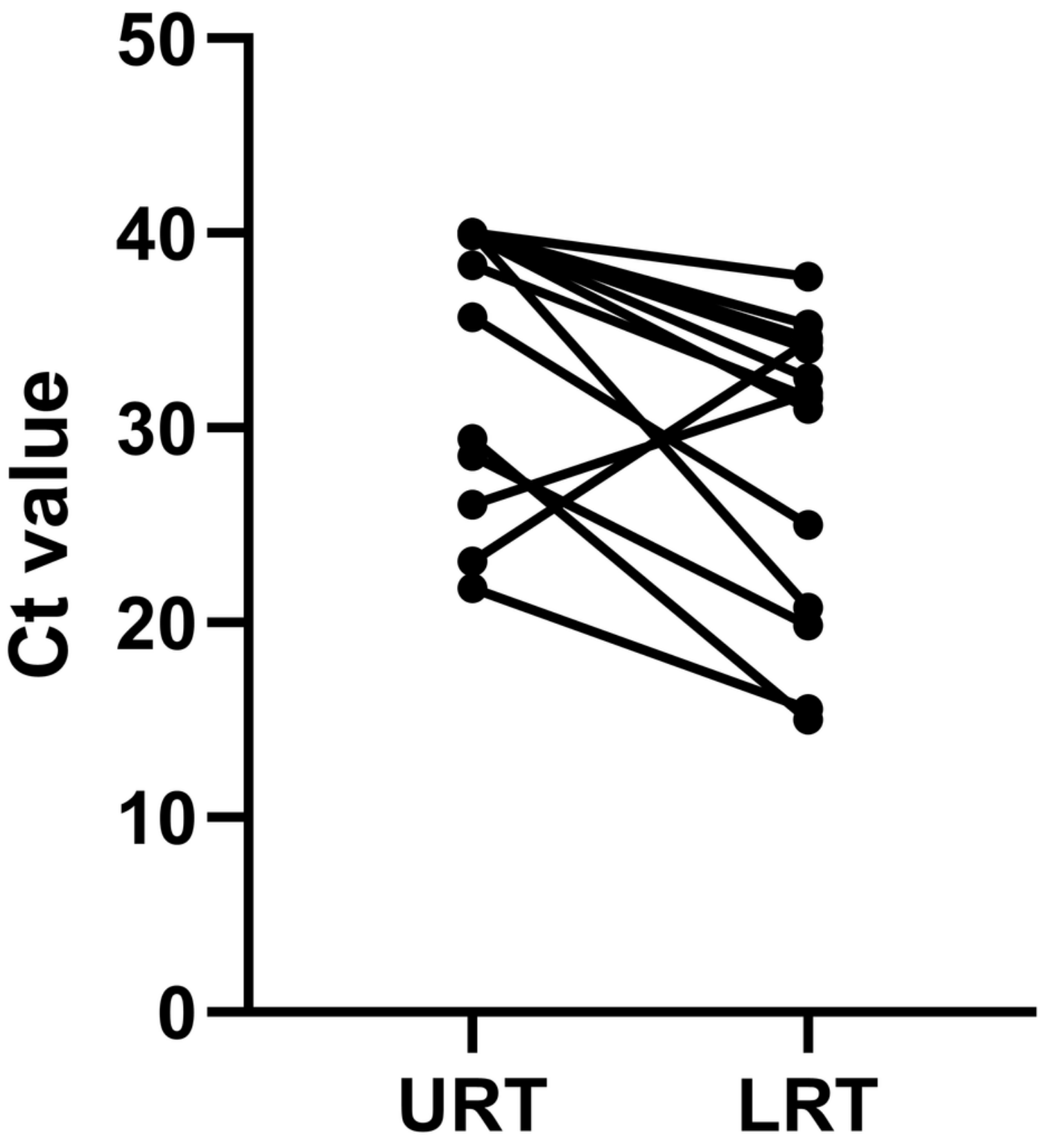

Figure 3

Comparison of the Ct value between URT and LRT samples upon admission to the ICU. URT, upper respiratory tract; LRT, lower respiratory tract; ICU, intensive care unit; $\mathrm{Ct}$, cycle threshold 


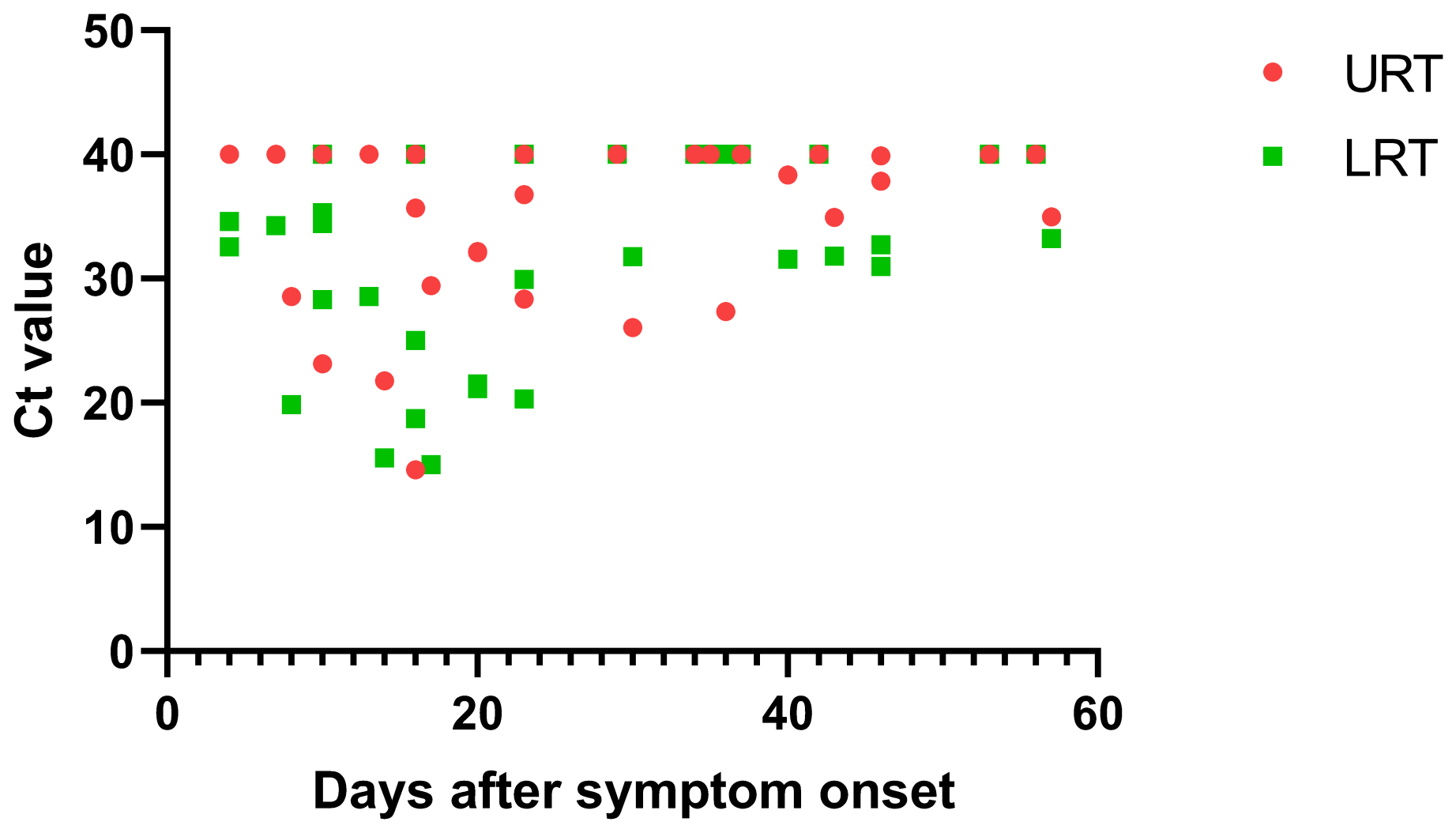

Figure 4

The relationship between Ct value and days after symptom onset in the URT and LRT samples. URT, upper respiratory tract; LRT, lower respiratory tract; ICU, intensive care unit; $\mathrm{Ct}$, cycle threshold 